\title{
Founder effect in prevalence of hereditary hearing loss in indigenous Siberian populations
}

\author{
V. Danilchenko ${ }^{1,2 *}$, M. Zytsar ${ }^{1,2}$, O. Posukh ${ }^{1,2}$ \\ ${ }^{1}$ Institute of Cytology and Genetics SB RAS, Novosibirsk, Russia \\ ${ }^{2}$ Novosibirsk State University, Novosibirsk, Russia \\ *e-mail: mikhalskaya@bionet.nsc.ru
}

Key words: hereditary hearing loss, founder effect, GJB2, RAI1, indigenous populations of Siberia

Motivation and Aim: Prevalence of many monogenic diseases can be determined by specific demographic and population factors (ethnic composition, migration, isolation, founder and bottleneck effects, proportion of consanguineous and assortative marriages). Nonsyndromic hearing loss (HL) is one of the most common monogenic disorders and several dozen genes contribute to its pathogenesis. It is well known that pathogenic variants in gene GJB2 (MIM 121011, 13q12.11) encoding connexin 26 (Cx26) account for a significant portion of hereditary HL and their spectrum and prevalence are highly specific for various populations. There are more limited data on prevalence of pathogenic variants in other genes associated with HL. We earlier found predominance of three major recessive GJB2 pathogenic variants (c.-23+1G $>$ A, c.235delC, p.W172C) in indigenous populations of Tuva and Altai and revealed common haplotypes for each of them that implies founder effect in their prevalence. Whole exome sequencing was applied to identifying candidate causal variants for undiagnosed hereditary HL cases in Altai [1]. Homozygous novel variant c.5254G>A in gene RAI1 (MIM 607642, 17p11.2) was revealed in association with $\mathrm{HL}$ in several Altaian families and its carrier frequency was estimated as $3.33 \%$ in Altaian control sample while c.5254G $>$ A was not found in Tuvinian patients and controls. This study aims to evaluate the role of founder effect in prevalence of major pathogenic variants in genes associated with nonsyndromic HL among indigenous populations of Siberia.

Methods and Algorithms: To investigate genetic background of pathogenic variant c.5254G $>$ A in the RAI1 gene, Sanger sequencing was applied for analysis of the RAII gene coding region encompassing exons 3, 4, 5 and part of exon 6 with flanking intronic regions in all Altaians homozygous or heterozygous for c.5254G $>$ A.

Results: All studied Altaian individuals with c.5254G $>$ A share a specific allele C-A$\mathrm{Q}_{13}\left[\mathrm{CAG}\right.$ CAA $\left.(\mathrm{CAG})_{10} \operatorname{del}(\mathrm{CAG}) \mathrm{CAA}\right]-\mathrm{G}-\mathrm{c} .5254 \mathrm{G}>\mathrm{A}$ that implies the common origin of this pathogenic variant in Altaians.

Conclusion: Identification of uniform allele bearing pathogenic variant c.5254G $>\mathrm{A}$ in gene RAII along with specific common genetic background for major pathogenic variants c. $-23+1 \mathrm{G}>\mathrm{A}$, c. $235 \mathrm{delC}$, p.W172C in gene GJB2 confirms important role of founder effect in prevalence of nonsyndromic HL among indigenous populations of Siberia.

Acknowledgements: Supported by the Project 0324-2018-0016 and by the RFBR (18-3400166_mol-a, 17-29-06016_ofi-m).

\section{References}

1. Churbanov A.Y. et al. (2016) Whole exome sequencing reveals homozygous mutations in RAI1, OTOF, and $S L C 26 A 4$ genes associated with nonsyndromic hearing loss in Altaian families (South Siberia). PLoS One. 11(4):e0153841. DOI 10.1371/journal.pone.0153841. 